\title{
A CURRENT-MODE LOGARITHMIC FUNCTION CIRCUIT
}

\author{
MUHAMMAD TAHER ABUELMA'ATTI* and OSAMA OGLAH FARIS \\ King Fahd University of Petroleum and Minerals, Box 203, Dhahran 31261, Saudi Arabia
}

(Received 18 October 2003; In final form 14 November 2003)

\begin{abstract}
A new current-mode analog circuit configuration that implements the function $\ln (x / y)$ is proposed. The circuit uses bipolar transistors and resistors and is suitable for integration. In the proposed circuit the ratio $(x / y)$ can be larger or smaller than unity. Simulation results are included.
\end{abstract}

Keywords: Function generator; Bipolar transistors

\section{INTRODUCTION}

Logarithmic functions are widely used in many applications; for example, instrumentation, telecommunication, neural networks, medical equipment, disk drives, and active filters. This justifies the continuing interest in developing logarithmic function circuits manifested by the relatively large number of publications in this area; see for example Refs. [1-19] and the references cited therein. Traditionally, a logarithmic function circuit, with input voltage and output voltage (or current) can be realized by exploiting to advantage the inherent exponential transconductance characteristic of the pn junction in a diode [12], bipolar transistor [5], or LED [16], to introduce the required nonlinearity into a circuit based on a current-feedback-operational [12], a transimpedance-amplifier [19], an operational-amplifier $[1,16]$, a transconductance-feedback-amplifier [18], or a current-conveyor [13]. Alternatively, a pseudo-logarithmic function can be implemented using piecewise linear approximations and cascaded limiting amplifiers [6]. This logarithmic function can be realized in GaAs hetrojunction bipolar transistor (HBT) technology [2,3], GaAs MESFET technology $[4,15]$, bipolar, CMOS or BiCMOS technology $[8-11,14]$. On the other hand, current mode circuits, with current inputs and current outputs, enjoy the attractive features of a wide current signal dynamic range even with low power supply voltages and wide bandwidths. Recently, a bipolar current-mode logarithmic function generator circuit has been reported [17]. This circuit can realize a logarithmic function of the form

$$
I_{\text {out }}=C \ln \frac{I_{\text {in } 1}}{I_{\text {in2 }}}
$$

\footnotetext{
* Corresponding author. E-mail: mtaher@kfupm.edu.sa
} 
where $C$ is a constant, $I_{\text {out }}$ is the output current, and $I_{\text {in } 1}$ and $I_{\text {in2 }}$ are the input currents. However, since $I_{\text {out }}$ must be positive, then the condition $I_{\text {in } 1}>I_{\text {in2 }}$ must be satisfied. Thus, the circuit cannot realize a true logarithmic function circuit where $I_{\text {in1 }}$ and $I_{\text {in2 }}$ can attain arbitrary positive values and $I_{\text {out }}$ can attain any positive or negative value. The major intention of this paper is, therefore, to present such a circuit.

\section{PROPOSED CIRCUIT}

The proposed circuit is shown in Figure 1. Ignoring the base-currents, for the translinear network formed of transistors $Q_{1}, Q_{2}$ and resistor $R_{1}$ we have the following relation:

$$
V_{\mathrm{BE} 2}=I_{\mathrm{o}} R_{1}+V_{\mathrm{BE} 1},
$$

where $I_{\mathrm{o}}$ is the current through resistor $R_{1}$. In Eq. (2), the base-emitter voltage $V_{\mathrm{BE} i}$ of the transistor $Q_{i}$ can be expressed as

$$
V_{\mathrm{BE} i}=V_{\mathrm{T}} \ln \left[\frac{I_{\mathrm{E} i}}{I_{\mathrm{S} i}}\right],
$$

where $V_{\mathrm{T}}$ is the thermal voltage, $I_{\mathrm{E} i}$ is the emitter current, and $\mathrm{I}_{\mathrm{S} i}$ is the saturation current of the transistor $Q_{i}$. Equation (3) is valid only if $I_{\mathrm{E} i} \gg I_{\mathrm{S} i}$, which is usually the case if the transistor $Q_{i}$ is biased in the active region. Combining Eqs. (2) and (3) and ignoring the base-currents, yields

$$
I_{\mathrm{o}}=\frac{V_{\mathrm{T}}}{R_{1}} \ln \left[\frac{I_{x}}{I_{y}}\right],
$$

where $I_{x}$ and $I_{y}$ are positive input currents.

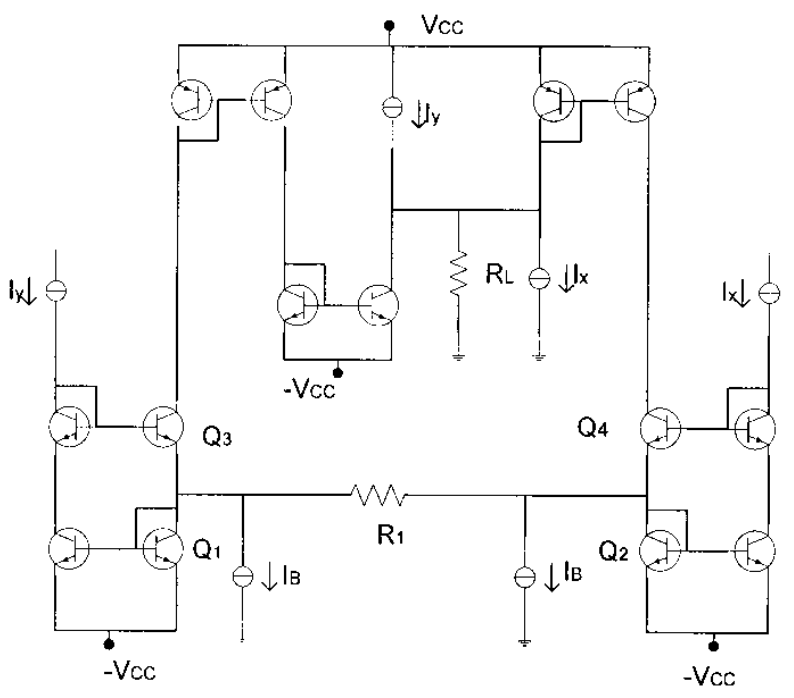

FIGURE 1 Proposed logarithmic circuit. 
Figure 1 and Eq. (4) show that $I_{\mathrm{o}}$ can be positive or negative. This sets no restriction on the ratio between the input currents $I_{x}$ and $I_{y}$ However, to maintain the positive emitter currents of the transistors $Q_{3}$ and $Q_{4}$ it is essential to ensure that $I_{\mathrm{o}}<I_{x}+I_{\mathrm{B}}$ and that $I_{\mathrm{o}}<I_{y}+I_{\mathrm{B}}$. This can be easily achieved by proper selection of the constant current $I_{\mathrm{B}}$.

In order to sense the current $I_{\mathrm{o}}$ and to force it through a grounded load resistor, additional current mirrors are introduced. Simple and straightforward analysis, ignoring the base-currents, shows that the current through the grounded resistor $R_{\mathrm{L}}$ is given by

$$
I_{\mathrm{L}}=2 I_{\mathrm{o}}
$$

\section{SIMULATION RESULTS}

The proposed circuit of Figure 1 was simulated using the ICAPS circuit simulation program. The transistors used for simulation are 2N2222 for npn transistors and 2N2905 for the pnp transistors. The output current was measured by sensing the voltages across a load resistor $R_{\mathrm{L}}=1.0 \mathrm{k} \Omega$. The results obtained with $I_{\mathrm{B}}=0.1 \mathrm{~mA}, R_{1}=2.6 \mathrm{k} \Omega$ and $V_{\mathrm{CC}}=3 \mathrm{~V}$ are shown in Figure 2. It appears from Figure 2 that the simulated results are in good agreement with the required function.

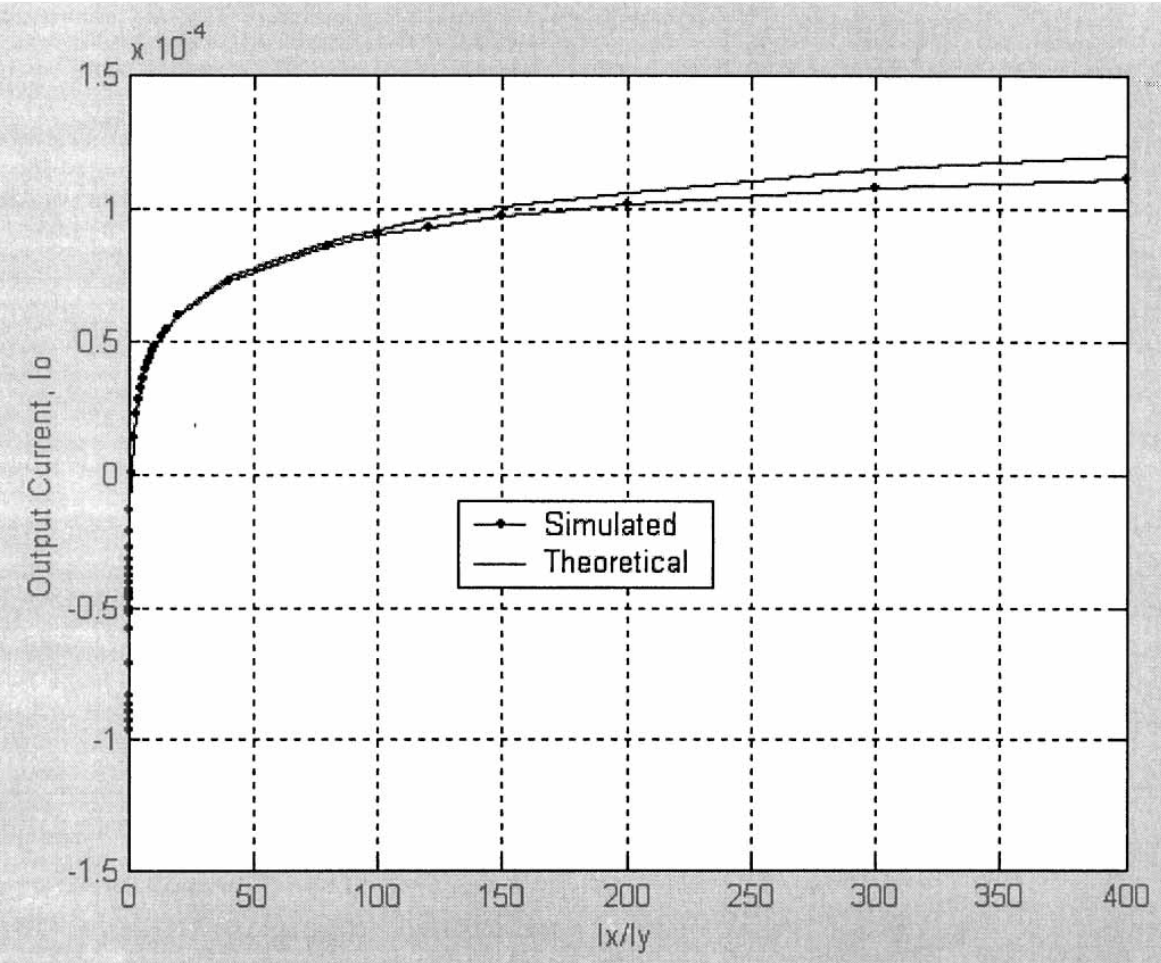

FIGURE 2 Calculated and simulated logarithmic function of Eq. (4). 


\section{DISCUSSION AND CONCLUSION}

In this paper a new circuit for realizing a logarithmic function has been presented. The proposed circuit of Figure 1 can realize a logarithmic function of the form of Eq. (1) for

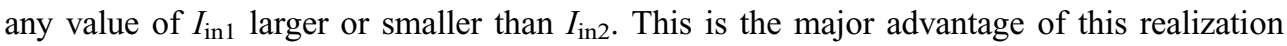
compared to previously published circuits, which require that $I_{\text {in } 1}$ is larger than $I_{\text {in2. }}$. The circuit uses only transistors, a single resistor and current sources, and is therefore attractive for integration.

The simulation results were obtained assuming that the ' $n$ ' parameters of the npn and pnp transistors are equal to unity. While this is, practically, a very good approximation, failure to meet this condition will result in an error. Another possible source of error is the effect of the collector-base voltage, through the base-width modulation (early effect) on the base-emitter voltage. Thus, transistors with large values of early voltage will result in better performance. Moreover, the base-emitter voltage is also affected by the finite ohmic resistances, especially the effective base resistance, and this will result in higher values of base-emitter voltage and may degrade the performance of the circuit of Figure 1. Finally, errors due to finite values of $\beta$ frequently degrade the performance of translinear circuits and the circuit of Figure 1 is not an exception. Thus, large values of $\beta$ would be preferable. Alternatively, many measures can be taken to minimize the effect of finite $\beta$ s on the performance of the circuit of Figure 1 [8].

\section{References}

[1] Grebene, A. B. (1984). Bipolar and MOS Integrated Circuit Design. John Wiley \& Sons, New York.

[2] Oki, A. K., Kim, M. E., Gorman, G. M. and Camou, J. B. (1988). High-performance GaAs hetrojunction bipolar transistor logarithmic IF amplifier. IEEE Microwave and Millimeter-wave Monolithic Circuits Symposium, pp. $41-45$.

[3] Oki, A. K., Kim, M. E., Gorman, G. M. and Camou, J. B. (1988). High-performance GaAs hetrojunction bipolar transistor logarithmic IF amplifier. IEEE T. Microw. Theory, 36, 1958-1965.

[4] Smith, M. A. (1988). A 0.5 to $4 \mathrm{GHz}$ true logarithmic amplifier utilizing monolithic GaAs MESFET technology. IEEE T. Microw. Theory, 36, 1986-1990.

[5] Wolffenbuttel, R. F. (1988). Digitally programmable accurate current sources for logarithmic control of the amplification or attenuation in a gain cell. IEEE J. Solid-St. Circ., 23, 767-773.

[6] Chadwick, P. E. (1989). Advances in logarithmic amplifiers. Proceedings of the Fifth International Conference on Radio Receivers and Associated Systems, pp. 51-58.

[7] Gertel, E., Johnson, D. M. and Kumar, M. (1990). 2-18 GHz Logarithmic amplification componentry. IEEE Microwave Theory and Techniques Symposium Digest, pp. 1093-1096.

[8] Kimura, K. (1992). Some circuit design techniques for bipolar and MOS pseudologarithmic rectifiers operable on low supply voltage. IEEE Transactions on Circuit Theory and Systems-I: Fundamental Theory and Applications, Vol. 39, pp. 771-777.

[9] Kimura, K. (1993). A CMOS logarithmic IF amplifier with unbalanced source-coupled pairs. IEEE J. Solid-St. Circ., 28, 78-83.

[10] Kimura, K. (1995). Circuit design techniques for very low-voltage analog functional blocks using triple-tail cells. IEEE Transactions on Circuit Theory and Systems-I: Fundamental Theory and Applications, Vol. 42, pp. 873-885.

[11] Kimura, K. (1996). Some circuit design techniques for low-voltage analog functional elements using squaring circuits. IEEE Transactions on Circuit Theory and Systems-I: Fundamental Theory and Applications, Vol. 43, pp. 559-576.

[12] Koli, K. and Halonen, K. (1996). Temperature compensated logarithmic peak detector. Analog Integr. Circ. S., 9 , 131-140.

[13] Bergouignan, F., Abouchi, N., Grisel, R., Caille, G. and Caranana, J. Designs of a logarithmic and exponential amplifiers using current conveyors. Proceedings of the Third International Conference on Electronics, Circuits and Systems, pp. 61-64.

[14] Koli, K. and Halonen, K. A $2.5 \mathrm{~V}$ temperature compensated CMOS logarithmic amplifier. IEEE Custom Integrated Circuits Conference, pp. 5.5.1-5.5.4.

[15] Acciari, G., Giannini, F. and Limiti, E. (1997). Theory and performance of parabolic true logarithmic amplifier. IEE Proceedings-Circuits Devices Systems, Vol. 144, pp. 223-228. 
[16] Acharya, Y. B. and Vyavahare, P. D. (2000). A low-current logarithmic LED electrometer. IEEE T. Instrum. Meas., 49, 5-9.

[17] Kumwachara, K. and Fujii, M. (2000). Low-voltage current mode power factor function. IEICE Transactions on Fundamentals, Vol. E83-A, pp. 172-178.

[18] Wilson, B. and Al-Gahtani, M. (2001). Improved logarithmic converter based on a transconductance feedback amplifier. Proceedings IEEE International Symposium on Circuits and Systems, pp. I651-I654.

[19] Hayes, M. J. (2002). A nonlinear optical preamplifier for sensing applications. IEEE Transactions on Circuit Theory and Systems-I: Fundamental Theory and Applications, Vol. 49, pp. 1-9. 

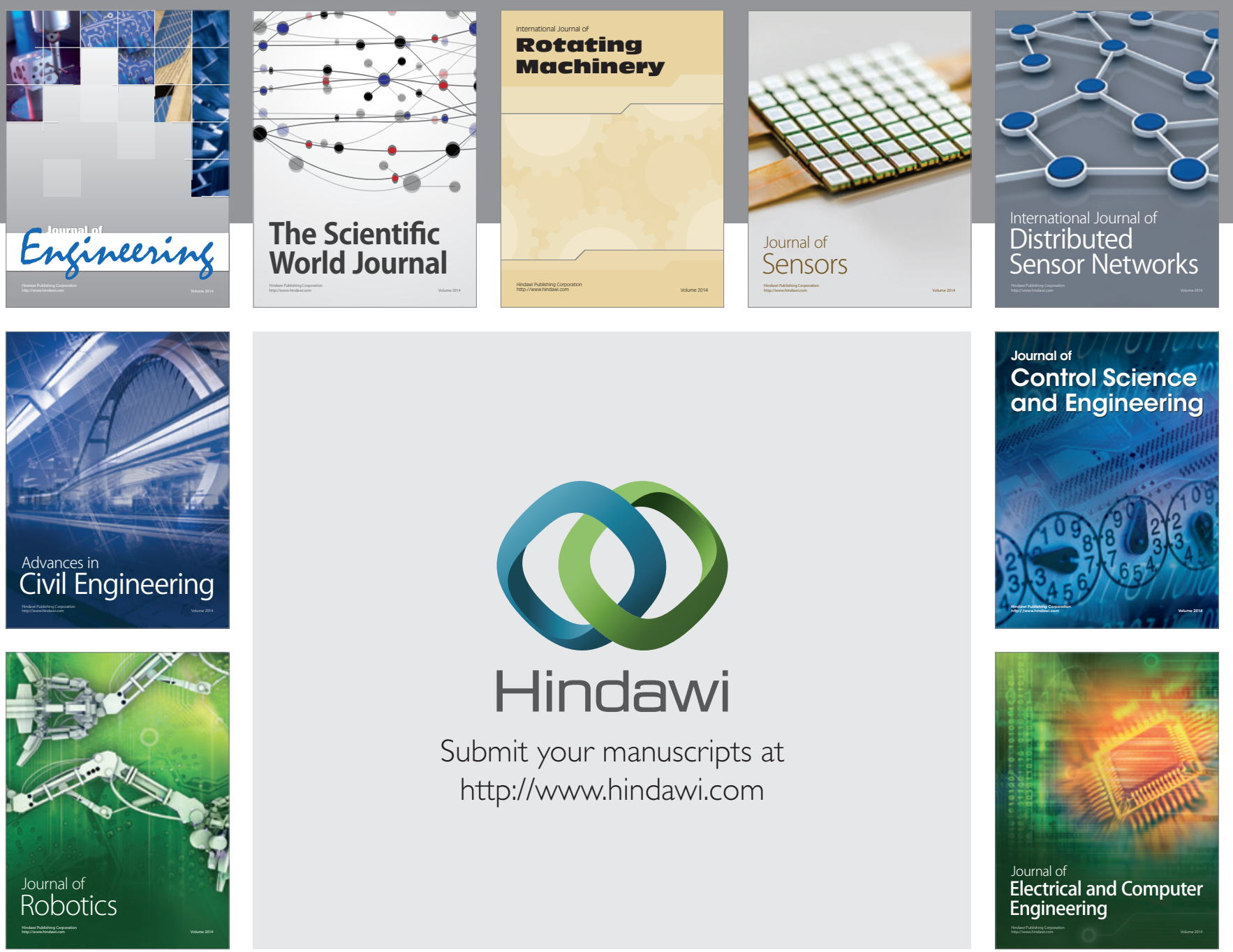

Submit your manuscripts at

http://www.hindawi.com
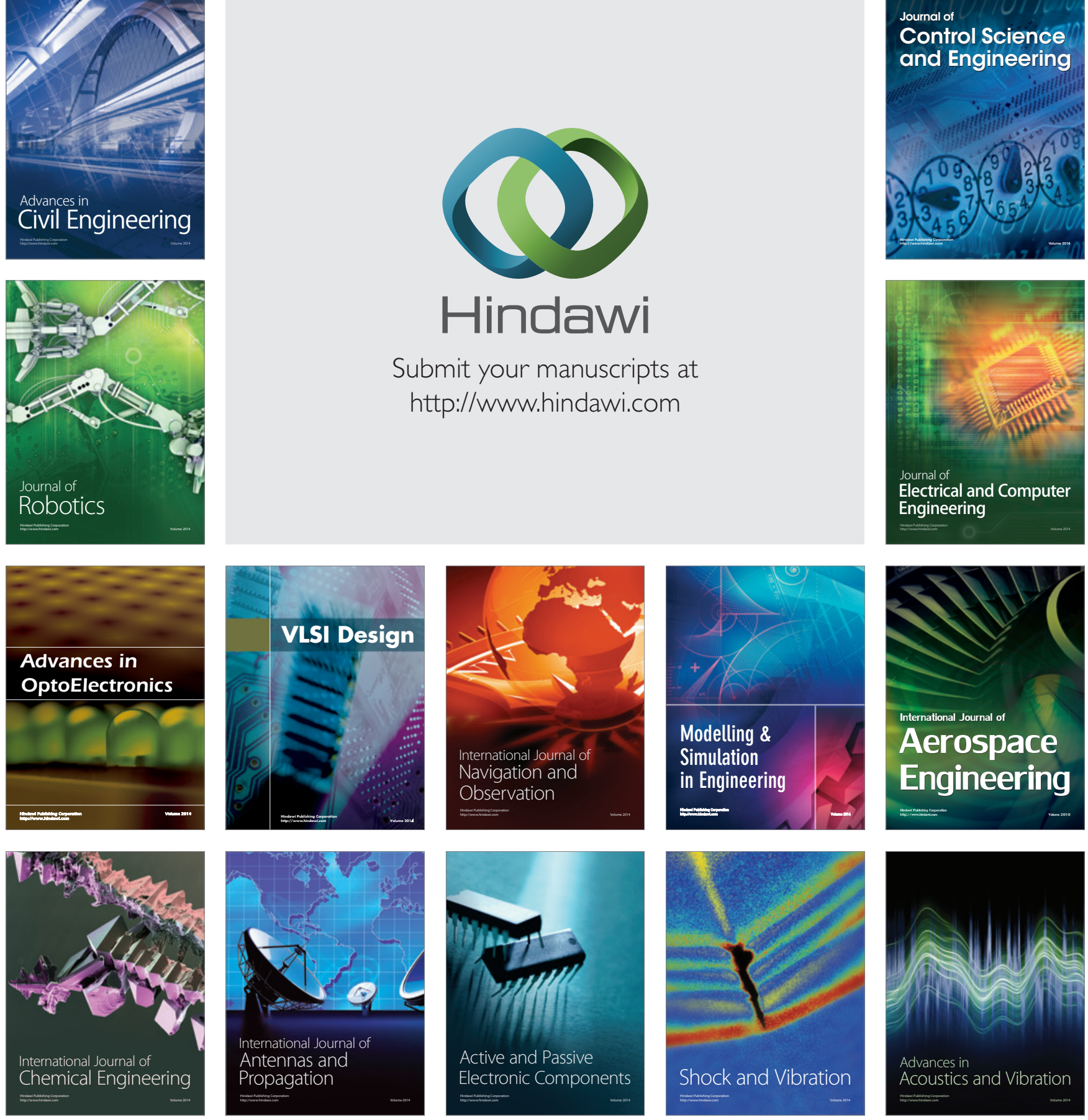